\title{
Reverberation Time and Power Model in Indoor Wireless Scenarios
}

\author{
Jun SHE, Yu YU, Peng-Fei CUI, Wen-Jun LU, Hong-Bo ZHU \\ Jiangsu Key Laboratory of Wireless Communications, Nanjing University of Posts and Telecommunications, \\ Xinmofan Street 66, 210003 Nanjing, China
}

\{2015010202,2013010203,2015010102,wjlu,wilab\}@njupt.edu.cn

Submitted November 25, 2017 / Accepted March 26, 2018

\begin{abstract}
A novel, room-electromagnetics-theory-based model for reverberation time, path gain and Power Delay Profile (PDP) is proposed. Unlike the traditional models describing only the reflections, the new model takes not only reflections at boundaries, but also the effects including scattering, diffraction and air absorption along the propagation path into consideration. Extensive measurements at 2.6 GHz under Line-Of-Sight (LOS) conditions are carried out not only in enclosed structures, but also in semi-enclosed scenarios which are normally with higher average absorptive coefficients. Hence, the application of reverberation model is extended compared to open literature. Reverberation time and path gain values predicted by the proposed model are in good agreement with these measurement results obtained in various indoor wireless environments. In addition, a novel PDP model with lower complexity is proposed based on measured path gain and Nakagami-m distribution. The proposed models are proved to be more accurate than traditional reverberation models.
\end{abstract}

\section{Keywords}

Indoor environment, reverberation time, path gain, Power Delay Profile (PDP), room electromagnetics

\section{Introduction}

Over the last decade, studies based on room electromagnetics manage to provide more insightful physical understanding for indoor wireless channels [1-7]. The room electromagnetics theory [1] points out that the electromagnetic waves in indoor wireless scenarios and the acoustic waves in room acoustics share similar behaviors in various aspects. The room electromagnetics theory explains the quasi-linear decaying of Power Delay Profile (PDP) in indoor scenarios [1], [4]. In reverberation models, the PDP is interpreted as the superposition of primary component and reverberant component. The former is subjected to power-law decaying while the latter experiences a quasi-linear decaying caused by reverberation effect [8]. Reverberation models use the reverberation effect instead of wave guiding effect to physically explain the phenomenon that path gain index falls far below 2 (i.e. index for free-space propagation) in indoor scenarios. The index describes the decaying of primary component in reverberation models, and it can get closer to 2 than in traditional models [4]. Reverberation time is a key parameter in reverberation models [5-7], [9], [10]. It originates from room acoustics, where it is defined as the time in which the total energy falls to one millionth of its initial value [11-13]. Hence, the modeling of reverberation time is of great importance, as it is a basis of reverberation models. Most reported electromagnetic reverberation time models are based on either Sabine's or Eyring's equation [1], [4-7], [11], [12]. In analogy with acoustics, it is proposed in the two equations that electromagnetic reverberation time in an indoor wireless scenario is solely dependent on three parameters that are fixed by propagation geometry and materials: volume, surface and average absorption coefficient of the environment [11-14]. In these reverberation time models, however, only the mechanism of specular reflections is taken into account, while the other effects are ignored. Validation of reverberation time models is rarely found in open literature, and in these limited validations, overestimates or underestimates are often observed by comparing the parameters obtained from propagation geometry, materials and the channel measurement data. Also, the small scale fading characteristics including the modeling of PDP are rarely investigated. Meanwhile, most of these studies are limited in enclosed environments like conference rooms and classrooms [1-7], but semi-enclosed scenarios are rarely studied.

Therefore, it is highly desired to develop a novel, accurate and more general reverberation model for indoor wireless scenarios. In this work, a new reverberation time and path gain model based on room electromagnetics is proposed. Unlike the traditional models describing only the reflections, the new model takes both the mechanism of reflections and effects of scattering, diffraction and air absorption along the propagation path into consideration. Reverberation parameters are obtained from both configuration of the scenarios and the channel measurement results in $2.6 \mathrm{GHz}$ indoor environments in the work. The proposed model is validated by the good agreement between pre- 
dicted and measured values and is proved to have better performance than conventional reverberation models. A novel model of PDP is also proposed using measured path gain values and the Nakagami-m distribution. Moreover, the model is applied in not only enclosed scenarios, but also semi-enclosed scenarios which are normally with higher average absorptive coefficients; the application of reverberation model is thus extended.

\section{Proposed Model}

In the following section, the model for electromagnetic reverberation time and path gain is proposed. Meanwhile, the acquisition of average absorption coefficient is presented.

\subsection{Electromagnetic Reverberation Time}

In this section, the electromagnetic reverberation time model is proposed. It is based on theories of room electromagnetics and room acoustics [1], [11-14]. The indoor scenario is geometrically modelled as system of image rooms where each reflection is presented by a narrow bundle of rays originating from the respective image source [12], [13]. In this case, the intensity of rays basically decreases proportionally as $(c t)^{-2}$, i.e. the free space loss. Meanwhile, the effects including non-specular reflections at walls (or ceilings, floors etc.), scattering, diffraction caused by objects, and the air absorption in the itinerary are taken into account by a path gain term $\exp (-m c t)$, which is dependent on an environmental factor $m$ and the travel distance $c t$ [13]. Moreover, the intensity of a ray bundle is reduced by a factor $1-a$ when it crosses a boundary of an image room $[12,13]$; if this happens $n$ times per second on the average, the intensity reduction due to wall absorption is $(1-\alpha)^{n t}$. Therefore, a reflection received at time $t$ has the average intensity

$$
I(t)=\frac{E_{0}}{4 \pi(c t)^{2}} \exp \{-[m c+n \ln (1-\alpha)] t\}
$$

where $I(t)$ is the intensity, $E_{0}$ is the energy generated by mirror sources at $t=0, V$ is the volume, $m$ is the environmental factor, $n$ is the average number of boundary crossing times per second, $\alpha$ is the average absorptive coefficient, $c$ is the light velocity in free space. Meanwhile, under the diffuse field assumption [1], [11-13], the average density of the reflections arriving at time $t$ can be approximated as [13]:

$$
r(t)=\frac{4 \pi c^{3} t^{2}}{V}
$$

where $r(t)$ is the average density of the reflections arriving at time $t$. Then, the time-dependent energy density can be derived by dividing the product of the last two expressions by light velocity [13]:

$$
\begin{aligned}
w(t) & =\frac{I(t) \cdot r(t)}{c} \\
& =w_{0} \exp \{-[m c+n \ln (1-\alpha)] t\}, \\
\text { with } w_{0}=\frac{E_{0}}{V} &
\end{aligned}
$$

where $w(t)$ is energy density at time $t, w_{0}$ is the energy density at $t=0 . n$ is the aforementioned average number of boundary crossing times per second. It can be approximated as [12]

$$
n=\frac{4 V}{c S}
$$

where $S$ is the total surface area. Hence, formula (3) can be re-written as follows:

$$
\begin{aligned}
w(t) & =w_{0} \exp \left\{-c t \frac{4 m V-S \ln (1-\alpha)}{4 V}\right\} \\
& =w_{0} \exp \left(-\frac{t}{T}\right) .
\end{aligned}
$$

The decaying exponent $T$ is defined as the reverberation time in an electromagnetic reverberation model. Hence, the reverberation time is proposed to be represented by the following formula:

$$
T=\frac{4 V}{c[4 m V-S \ln (1-\bar{\alpha})]} .
$$

\subsection{Average Absorption Coefficient}

In this section, the acquisition of absorption coefficient is presented [13], [15], [16]. As is mentioned above, the average absorptive coefficients of an indoor scenario can be calculated from the dimensions and reflective coefficients of materials. The electromagnetic property of a particular material in the investigated frequency range is presented by its permittivity $\varepsilon$. Fresnel formulas [15] at the air-dielectric interface are applied to obtain the reflection coefficients:

$$
\begin{gathered}
\Gamma_{\|}=\frac{-\varepsilon_{\mathrm{r}} \sin \theta+\sqrt{\varepsilon_{\mathrm{r}}-\cos ^{2} \theta}}{\varepsilon_{\mathrm{r}} \sin \theta+\sqrt{\varepsilon_{\mathrm{r}}-\cos ^{2} \theta}}, \\
\Gamma_{\perp}=\frac{\sin \theta+\sqrt{\varepsilon_{\mathrm{r}}-\cos ^{2} \theta}}{\varepsilon_{\mathrm{r}} \sin \theta+\sqrt{\varepsilon_{\mathrm{r}}-\cos ^{2} \theta}}
\end{gathered}
$$

where $\varepsilon_{\mathrm{r}}=\varepsilon_{2} / \varepsilon_{1}$, as $\varepsilon_{2}$ is the relative permittivity of a material, and $\varepsilon_{1}$ stands for relative permittivity of the air, $\theta$ is the angle of incident of the electromagnetic wave, $\Gamma_{\|}$and $\Gamma_{\perp}$ are the reflection coefficients for parallel and perpendicular polarizations, respectively. Meanwhile, the percentage of electromagnetic waves absorbed on material $i$ per unit area, denoted as $\alpha_{i}(f)$, can be determined by the following integral $[13,15]$ : 


$$
\alpha_{i}(f)=\int_{0}^{\frac{\pi}{2}}\left\{1-\frac{1}{2}\left[\left|\Gamma_{\|, i}(\theta, f)\right|^{2}+\left|\Gamma_{\perp, i}(\theta, f)\right|^{2}\right]\right\} \cos \theta \sin \theta \mathrm{d} \theta
$$

where $\alpha_{i}(f)$ is the percentage of absorption, $f$ is the frequency. With (8) - (10), the overall average absorption coefficient $\bar{\alpha}(f)$ of an indoor scenario is yielded:

$$
\bar{\alpha}(f)=\frac{\sum_{i} S_{i} \alpha_{i}(f)+S_{\mathrm{o}}}{S}
$$

where $\bar{\alpha}(f)$ is the overall average absorption coefficient, $S_{i}$ is the total surface area of a certain material $i$. For instance, material 1 is concrete, $S_{1}$ is the sum of surface areas of ceiling, walls and floor that are made of concrete, $\alpha_{1}(f)$ denotes the percentage of electromagnetic waves absorbed on concrete per unit area. The open area at the boundary of a scenario is considered as totally absorptive material, because the electromagnetic wave will not return when it leaves the scenario across the open area. Hence, $\alpha_{\mathrm{o}}(f)$ for open area equals to $1 . S_{\mathrm{o}}$ is the total surface area of the open area (the sum of surface areas of opened doors, etc.) at the boundary of scenario. $S_{\mathrm{o}} \alpha_{\mathrm{o}}(f)$ can thus be simplified as $S_{\mathrm{o}}$. The human presence, which will result in a decrease of reverberation time in indoor scenarios, must also be taken into consideration [16]. To this end, the influence of human presence is included in the model using effective coefficients:

$$
(S \alpha)_{\mathrm{eff}}=k \alpha_{\mathrm{hb}}(f) \cdot B S A .
$$

The presence of human body is treated as an additional material emerged in the scenario where $(S \alpha)_{\text {eff }}$ is the effective coefficient, $\alpha_{\mathrm{hb}}(f)$ is the effective absorptive coefficient of human body per unit area, and $k$ is the number of persons present in the scenario, BSA stands for Body Surface Area. When there are $k$ persons in the scenario, equation (11) becomes:

$$
\begin{aligned}
\bar{\alpha}(f) & =\frac{\sum_{i} S_{i} \alpha_{i}(f)+S_{\mathrm{o}}+(S \alpha)_{\mathrm{eff}}}{S} \\
& =\frac{\sum_{i} S_{i} \alpha_{i}(f)+S_{\mathrm{o}}+k \alpha_{\mathrm{hb}}(f) \cdot B S A}{S} .
\end{aligned}
$$

Hence, boundary materials, open area, and human presences can all be included in the proposed model using the arithmetic mean of absorption coefficients with the respective areas as weighting factor. With (5)-(13), the model for electromagnetic reverberation time is proposed.

\subsection{Path Gain and Power Delay Profile}

Models characterizing the power properties of wireless channel are presented in this section. The path gain model is based on [1], [4] and the PDP model is based on existing theories of Discrete Tapped Delay Line (DTDL) models [8], [17]. Reverberation parameters can be applied to obtain the distance-dependent path gain model as follows [1], [4]:

$$
G(d)=G_{0}\left(\frac{d_{0}}{d}\right)^{n}+G_{0, \mathrm{rev}} T \exp \left(-\frac{d}{c T}\right)
$$

where $d$ is the distance, $G(d)$ is the path gain at $d, n$ is the decaying exponent for the primary component, $T$ is the aforementioned reverberation time, $G_{0}$ and $G_{0 \text {,rev }}$ are reference path gains at the reference distance $d_{0}$.

So far, large scale fading parameters of the channels are characterized. Next, small scale fading parameters are analyzed to fully investigate the channels and obtain the PDP models. To this end, the DTDL method [8] is applied, the amplitude of each delay pin at each measurement position is normalized by the predicted path gain as follows:

$$
a_{m}\left(d, \tau_{k}\right)=\frac{A_{m}\left(d, \tau_{k}\right)}{\sqrt{G(d)}}
$$

where $A_{m}\left(d, \tau_{k}\right)$ is the amplitude at propagation delay $\tau_{k}$ and distance $d . \tau_{k}$ are the delay bins in the DTDL model, where $k=0,1,2, \ldots, N$. Random variables $a_{m}\left(d, \tau_{k}\right)$ in such scenarios are proposed to be expressed as Nakagami distributions [8], [17]:

$$
a_{m}\left(d, \tau_{k}\right) \sim \operatorname{Nakagami}\left(\mu\left(d, \tau_{k}\right), \omega\left(d, \tau_{k}\right)\right)
$$

where $\mu\left(d, \tau_{k}\right)$ and $\omega\left(d, \tau_{k}\right)$ denote the shape parameter and the scale parameter, respectively. The shape parameter represents the severity of the fading and it follows the lognormal distribution [8], [17], while the scale parameter describes the mean power of the normalized amplitudes averaged over a location [8]. It is proposed in the novel model that $\omega$ is distance-independent and it experiences a quasi-linear decaying versus the time delay $\tau$, for the reason of the aforementioned reverberation effect. The two parameters can be presented as follows:

$$
\begin{gathered}
\mu\left(d, \tau_{k}\right) \sim L N\left(m_{\mu}, \sigma_{\mu}\right), \\
W\left(d, \tau_{k}\right)=B_{\omega} \tau_{k}+C+z,
\end{gathered}
$$

$W\left(d, \tau_{k}\right)$ is the scale parameter in $\mathrm{dB}, m_{\mu}, \sigma_{\mu}, B_{\omega}$ and $C$ are constants to fit, $z$ are normally distributed random variables.

Finally, the PDP model is obtained as:

$$
P\left(d, \tau_{k}\right)=\sum_{k=0}^{N}[\underbrace{\sqrt{G(d)}}_{\begin{array}{c}
\text { Large Scale } \\
\text { Fading }
\end{array}} \underbrace{a_{m}\left(d, \tau_{k}\right)}_{\begin{array}{c}
\text { Small Scale } \\
\text { Fading }
\end{array}}]^{2} \underbrace{\delta[\tau-(k-1) \Delta \tau]}_{\text {Delay Bins }} .
$$

$P\left(d, \tau_{k}\right)$ is the modeled PDP, it is composed of a series of delay bins. $G(d)$ is the path gain value at distance $d$, it characterizes the large scale fading mechanism in the scenario. $a_{m}\left(d, \tau_{k}\right)$ represents the normalized amplitude of each tap at each location, it changes with small spatial variations in the channel. $\delta[\tau-(k-1) \Delta \tau]$ represents the delay bins with step size $\Delta \tau$.

It is concluded that the complexity of fitting and modeling procedures are reduced in the proposed model compared to those in literature [8], [17]. 
In the proposed model, it can be summarized that model parameters including volumes, surface areas, absorption coefficients, total surface areas and average absorption coefficients are fixed by propagation geometry and materials. Path gain at different distances, measured PDP and SPDP, decaying slopes, normalized amplitudes are obtained from channel measurement data. Environmental factor $m$, path gain model parameters including decaying exponent $n$ and reference path gains $G_{0}$ and $G_{0, \text { rev }}$, PDP model parameters $m_{\mu}, \sigma_{\mu}, B_{\omega}$ and $C$ are obtained by fitting.

\section{Measurements and Data Processing}

\subsection{Measurement Campaigns}

The three scenarios are shown in Fig. 1. Channel measurements are firstly performed in scenario A, a rectangular meeting room furnished with a conference table and a few chairs. One side of the room is furnished with windows [18]. Then, in scenario B, a typical corridor in an office building is studied. As is illustrated in Fig. 1, the open area is considered as totally absorptive material. Panels and elevator doors are made of metal. In scenario C, a stairwell is subsequently investigated using the same approach. The step height is $0.17 \mathrm{~m}$. The big door beside the elevator in B is open when measuring while all other doors in these scenarios are closed. In these scenarios, LOS environments are studied.

The measurement is conducted by a vector network analyzer Agilent $8753 \mathrm{ES}$, which generates a 10-dBm signal sweeping from 2.5 to $2.69 \mathrm{GHz}$. 50 sweeps are successively carried out with a sweep period of $400 \mathrm{~ms}$ for one Tx-Rx position and at least 201 points in each sweep. Identical omni monopole antennas with $3 \mathrm{dBi}$ gain are used as Tx and Rx antennas. In scenario A and C, the $2 \times 2$ MIMO measurements are carried out by two RF switch matrices Agilent 34980A. In particular, both VV-VV and HH-VV polarizations are investigated in scenario $\mathrm{C}$. The same monopole antennas are used for both vertical polarization
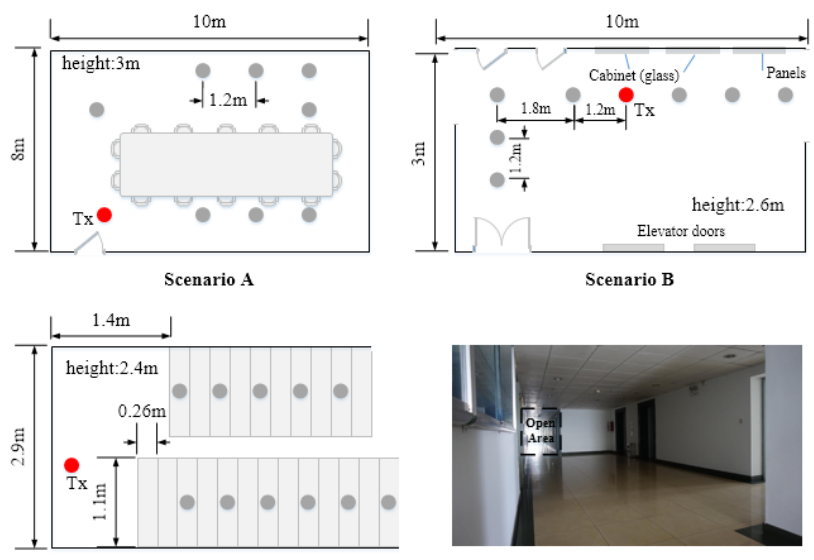

Scenario $\mathrm{C}$

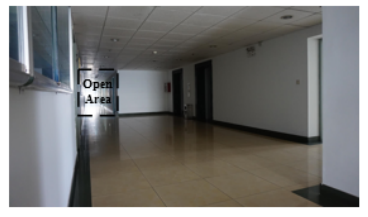

Photograph
(Scenario B)

Fig. 1. Configuration of scenarios A-C and a photograph of B.

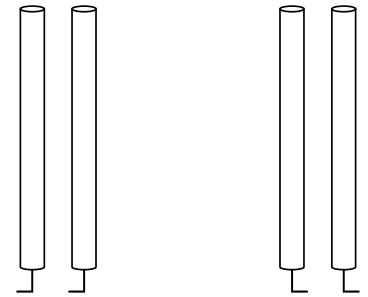

VV - VV

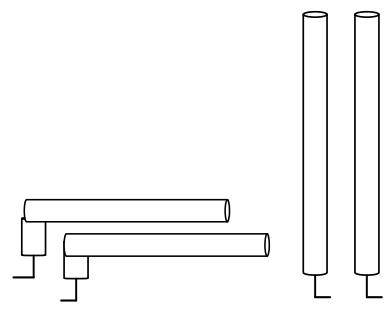

$\mathrm{HH}-\mathrm{VV}$

Fig. 2. Illustration of the different polarizations.

and horizontal polarization. The monopole-pattern is controlled by a switch, as is illustrated in Fig. 2. The noise floor of the system is $-110 \mathrm{dBm}$. The measurements are performed at off-hour so that the channel is assumed to be time-invariant [19].

\subsection{Data Processing}

Main materials present in the scenarios are listed in Tab. 1, with their surface areas derived from the geometrical measurements and their absorption coefficients calculated with (8)-(10). Complex permittivity for materials and air at $2.6 \mathrm{GHz}$ is obtained from [15]. Metal is approximated as a perfect reflective material, and the open areas in B and $\mathrm{C}$ are equivalent to $100 \%$ absorptive material, as illustrated in Fig. 1. Hence, the average absorption coefficients can be obtained, as is shown in Tab. 2. The instantaneous PDP can be obtained from measurement data, using the same method as in [19]. The PDP is time-averaged [19] and subsequently spatial-averaged [4] to provide more insightful understandings on characteristics of the channel. Reverberation time can thus be determined from the slope of the quasi-linear tail of Spatial-averaged PDP (SPDP) [5]:

$$
\tau=-\frac{10 \log (\mathrm{e})}{\text { slope }}
$$

where $\tau$ is the reverberation time determined from measured SPDP, e is Euler's number. The obtained reverberation time values in the three scenarios are presented in Tab. 2.

\begin{tabular}{|c|c|c|c|c|c|}
\hline Scenario & $\begin{array}{c}\text { Concrete } \\
{\left[\mathbf{m}^{2}\right]} \\
\boldsymbol{a}=\mathbf{0 . 3 9}\end{array}$ & $\begin{array}{c}\text { Wood } \\
{\left[\mathbf{m}^{\mathbf{2}}\right]} \\
\boldsymbol{a}=\mathbf{0 . 4 6}\end{array}$ & $\begin{array}{c}\text { Glass } \\
{\left[\mathbf{m}^{\mathbf{2}}\right]} \\
\boldsymbol{a}=\mathbf{0 . 4 0}\end{array}$ & $\begin{array}{c}\text { Metal } \\
{\left[\mathbf{m}^{2}\right]}\end{array}$ & $\begin{array}{c}\text { Open Area } \\
{\left[\mathbf{m}^{\mathbf{2}}\right]}\end{array}$ \\
\hline $\mathrm{A}$ & 158 & 80 & 30 & - & - \\
\hline $\mathrm{B}$ & 78.9 & 28.8 & 3.2 & 3.7 & 13 \\
\hline $\mathrm{C}$ & 46.9 & - & - & - & 32.3 \\
\hline
\end{tabular}

Tab. 1. Surface areas of main materials present in the scenarios. 


\begin{tabular}{|c|c|c|c|c|c|c|c|c|c|}
\hline Scenario & Environment & Polarization & $V\left[\mathbf{m}^{3}\right]$ & $S\left[\mathbf{m}^{2}\right]$ & $k$ & $\bar{\alpha}$ & $\begin{array}{c}\text { Decaying slope } \\
{[\mathrm{dB} / \mathrm{ns}]}\end{array}$ & $\begin{array}{l}\text { Meas. } \\
\tau[\mathrm{ns}]\end{array}$ & $\mathrm{f}(\bar{\alpha})_{m}$ \\
\hline \multirow{3}{*}{ A } & \multirow{3}{*}{$\begin{array}{l}\text { Meeting room } \\
\text { (enclosed) }\end{array}$} & \multirow{3}{*}{ VV-VV } & \multirow{3}{*}{240} & \multirow{3}{*}{268} & 0 persons & 0.412 & 0.160 & 27.14 & 0.439 \\
\hline & & & & & 2 persons & 0.415 & 0.163 & 26.64 & $\overline{0.447}$ \\
\hline & & & & & 6 persons & 0.420 & 0.167 & 26 & 0.458 \\
\hline B & $\begin{array}{l}\text { Corridor } \\
\text { (enclosed) }\end{array}$ & $\mathrm{V}-\mathrm{V}$ & 79 & 130.6 & 0 persons & 0.464 & 0.256 & 16.99 & 0.474 \\
\hline \multirow{3}{*}{ C-1 } & \multirow{3}{*}{$\begin{array}{c}\text { Stairwell } \\
\text { (semi-enclosed) }\end{array}$} & \multirow{3}{*}{ VV-VV } & \multirow{3}{*}{42.9} & \multirow{3}{*}{79.2} & 0 persons & 0.644 & 0.628 & 6.92 & 1.042 \\
\hline & & & & & 2 persons & 0.652 & 0.692 & 6.27 & 1.141 \\
\hline & & & & & 6 persons & 0.670 & 0.669 & 6.49 & 1.085 \\
\hline \multirow{3}{*}{$\mathrm{C}-2$} & \multirow{3}{*}{$\begin{array}{c}\text { Stairwell } \\
\text { (semi-enclosed) }\end{array}$} & \multirow{3}{*}{ HH-VV } & \multirow{3}{*}{42.9} & \multirow{3}{*}{79.2} & 0 persons & 0.644 & 0.603 & 7.19 & 1.002 \\
\hline & & & & & 2 persons & 0.652 & 0.636 & 6.83 & 1.046 \\
\hline & & & & & 6 persons & 0.670 & 0.662 & 6.57 & 1.071 \\
\hline
\end{tabular}

Tab. 2. Reverberation parameters of measured scenarios.

\section{Validation and Discussion}

\subsection{Overview of Traditional Models}

To further discuss the performances of traditional reverberation models in scenarios, the prediction using traditional models is studied in preliminary. A number of experimental analysis using room electromagnetics can be found in existing studies. Here, studies with sufficient data for reverberation time values, propagation geometry and materials in open literature are re-investigated as supplementary [7], [20-22]. These data are processed using the following functions:

$$
f(\bar{\alpha})_{m}=\frac{4 V}{c S \tau}
$$

and

$$
\left\{\begin{array}{l}
\mathrm{f}(\bar{\alpha})_{\text {Sabine }}=\bar{\alpha}, \\
\mathrm{f}(\bar{\alpha})_{\text {Erying }}=-\ln (1-\bar{\alpha}) .
\end{array} .\right.
$$

In (21), (22), functions of $\mathrm{f}(\bar{\alpha})$ are presented. It is noted that $\mathrm{f}(\bar{\alpha})_{m}$ in (21) is derived from the measurement, and (22) represents the predicted $\mathrm{f}(\bar{\alpha})$ values according to existing models. The first formula in (21) stands for models based on Sabine's equation [11], while the second formula represents the models based on Eyring's equation [12]. In Fig. 3, curves of the two types of models are presented in color. With the data in scenario A-C and the open literature, an integrated overview is finally determined, as shown in Fig. 3. Circles stand for measured values for enclosed and semi-enclosed scenarios in this work. Open literature data, most of which are obtained in enclosed structures, are illustrated by asterisks. Firstly, it is found that semi-enclosed scenarios B and C achieve higher $\bar{\alpha}$ than enclosed scenarios in available references. The lowest $\bar{\alpha}$ is obtained with [21], where measurement data are collected in the NLOS scenario with a high reflective metal object present between Tx and Rx. Apart from [21], all other $\bar{\alpha}$ falls between 0.39 and 0.41 . This can be attributed to the similarity of materials and configurations among enclosed indoor scenarios, knowing that the absorption coefficient of concrete and glass are around 0.40 in the range of 1 to $10 \mathrm{GHz}$, as calculated with the aforementioned formulas. The performance of the models can be investigated by fitting the curves using $\left(\bar{\alpha}, \mathrm{f}(\bar{\alpha})_{m}\right)$ of different scenarios, with $\bar{\alpha}$ deter-

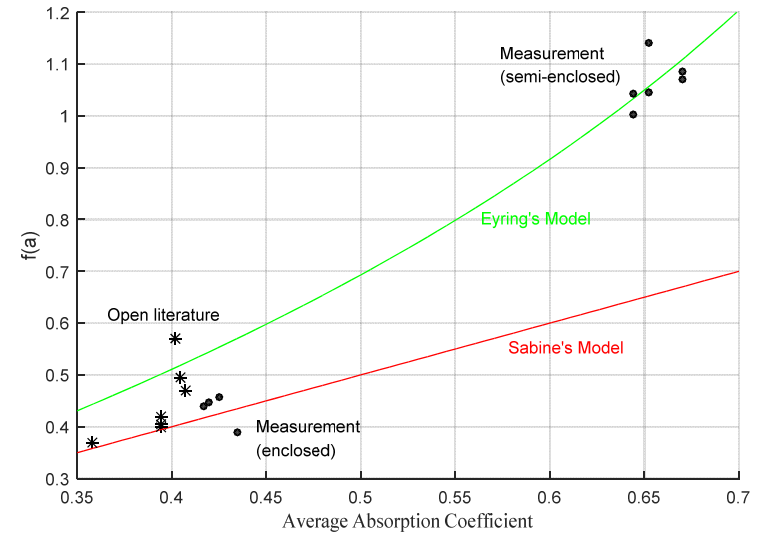

Fig. 3. Overview of reverberation models' performance.

mined from propagation geometry and materials, and $\mathrm{f}(\bar{\alpha})_{m}$ obtained from channel measurements. It is observed that models based on Sabine's equation fail to provide correct results for higher absorptive scenarios and that both models make quite a few errors in predicting the reverberation time.

\subsection{Validation of Reverberation Time Models}

In this section, the proposed model is applied to predict reverberation time and validated by comparing the predicted values with measurement results and other reverberation models. Firstly, the environmental factor $m$ of the proposed model is obtained by fitting using the measured reverberation time values. The measured reverberation time values were obtained by the measured SPDP and (20). Main procedures to obtain $m$ are illustrated in the diagram in Fig. 4. Then, the proposed model in the three different scenarios is presented in Fig. 5. For A and C, results with the presence of 0,2 and 6 persons are presented. For $C$, the curves for different polarizations are shown in the same graph for the purpose of comparison. The blue points represent the measured reverberation time $\tau$ obtained from SPDP. The grey points show the time-averaged PDP at different measurement distances. Prediction based on Sabine's and Eyring's equation is colored in red and green, respectively. The black curve stands for the prediction by the proposed model. It is clearly observed that for most cases, good agreement between predicted and measured values is obtained by using the proposed model. 


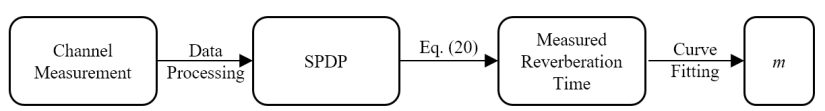

Fig. 4. Procedures to obtain the environment factor.

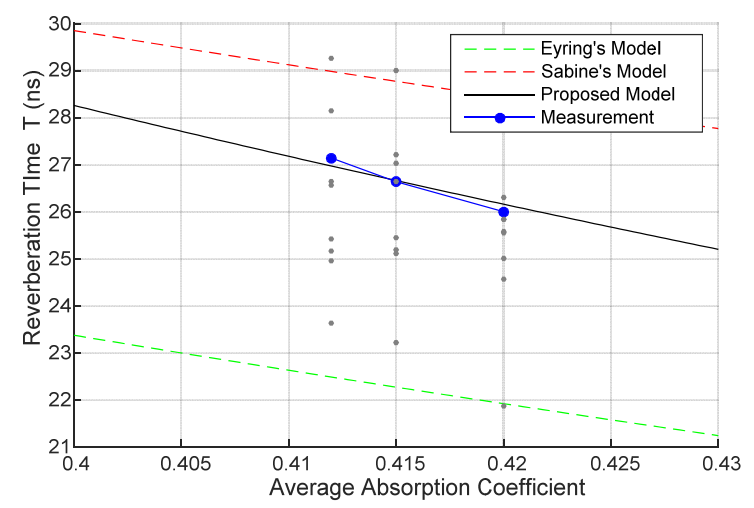

(a) Scenario A

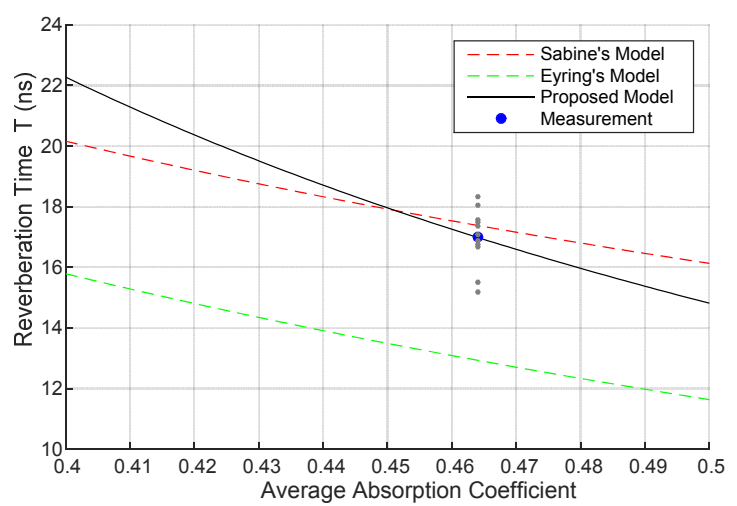

(b) Scenario B

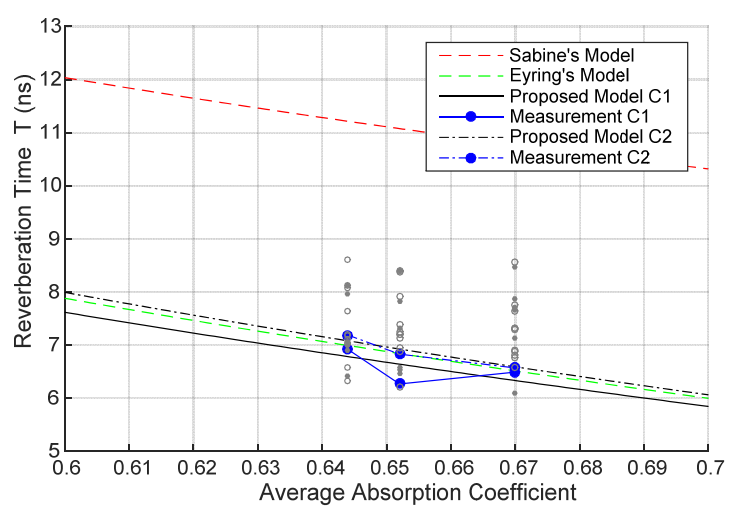

(c) Scenario C

Fig. 5. Reverberation time values from measurements and predictions in 3 scenarios.

\begin{tabular}{|c|c|c|c|c|}
\hline \multirow{2}{*}{ Scenario } & \multirow{2}{*}{$\boldsymbol{m}(\mathbf{d B} / \mathbf{m})$} & \multicolumn{3}{|c|}{ RMSE } \\
\cline { 3 - 5 } & & Sabine's & Eyring's & Proposed \\
\hline $\mathrm{A}$ & -16.1 & 3.32 & 3.91 & 1.76 \\
\hline $\mathrm{B}$ & -12.1 & 0.99 & 4.17 & 0.94 \\
\hline $\mathrm{C} 1$ & -18.3 & 9.79 & 0.92 & 0.95 \\
\hline $\mathrm{C} 2$ & -22.3 & 3.75 & 0.83 & 0.78 \\
\hline
\end{tabular}

Tab. 3. RMSE of predictions using different reverberation models.
Values of environmental factor $m$, and the Root Mean Square Error (RMSE) between predictions and measured results based on different reverberation models are presented in Tab. 3. One can conclude from Fig. 5 and Tab. 3 that the proposed model has better performance than the traditional reverberation models. It is interesting to note that reverberation time values using different polarizations for scenario $\mathrm{C}$ is presented by only one function using Eyring's or Sabine's equation, but they can be modeled separately by the proposed model. This can be attributed to that only reflections are taken into account in these models, but the other effects which electromagnetic waves experience in itinerary such as the depolarizations could not be presented.

\subsection{Validation of Path Gain Models}

In this section, the proposed model is validated by fitting with measured path gain values at different $T x-R x$ distances.

In Fig. 6, the proposed model and measured path gain values are presented. Model parameters that are obtained by fitting are presented in Tab. 4. For C-1, results for up and down direction are separately fitted because of their distinct behaviors. It is found that the fitted curves based on the proposed model are in good agreement with measured path gains. Meanwhile, human presence is proved to have generally much smaller impact on the received power than the distance in such indoor environments.

\begin{tabular}{|c|c|c|c|c|}
\hline Scenario & $\begin{array}{c}\text { Human } \\
\text { Presence }\end{array}$ & $\boldsymbol{n}$ & $\boldsymbol{G}_{\mathbf{0}}(\mathbf{d B})$ & $\boldsymbol{G}_{\mathbf{0}, \text { rev }}(\mathbf{d B})$ \\
\hline \multirow{4}{*}{ A } & 0 person & 3.184 & -36.1 & 26.7 \\
\cline { 2 - 5 } & 2 persons & 1.989 & -54.6 & 16.9 \\
\cline { 2 - 5 } & 6 persons & 1.818 & -53.7 & 0.8 \\
\hline B & 0 person & 1.807 & -29.7 & 24.3 \\
\hline \multirow{3}{*}{$\begin{array}{c}\text { C-1 } \\
\text { Up }\end{array}$} & 0 person & 2.430 & -38.3 & 26.7 \\
\cline { 2 - 5 } & 2 persons & 2.595 & -37.2 & 12.7 \\
\cline { 2 - 5 } & 6 persons & 2.536 & -37.6 & 25.9 \\
\hline \multirow{3}{*}{$\begin{array}{c}\text { C-1 } \\
\text { Down }\end{array}$} & 0 person & 3.272 & -45.2 & 27.2 \\
\cline { 2 - 5 } & 2 persons & 4.133 & -43.3 & 26.6 \\
\cline { 2 - 5 } & 6 persons & 3.806 & -46.1 & 26.2 \\
\hline \multirow{3}{*}{ C-2 } & 0 person & 1.248 & -54.5 & 5.3 \\
\cline { 2 - 5 } & 2 persons & 1.656 & -53.4 & 0.3 \\
\cline { 2 - 5 } & 6 persons & 1.501 & -55.0 & 16.8 \\
\hline
\end{tabular}

Tab. 4. Path gain model parameters.

\subsection{Validation of PDP Models}

In this section, the proposed PDP model is validated. The data in use and the model parameters are shown in Tab. 4. As shown in Tab. 5, the first four parameters are chosen to validate the model. $m_{\mu}$ and $\sigma_{\mu}$ were determined by log-normal fitting, $B_{\omega}$ and $C$ were determined by linear fitting. 


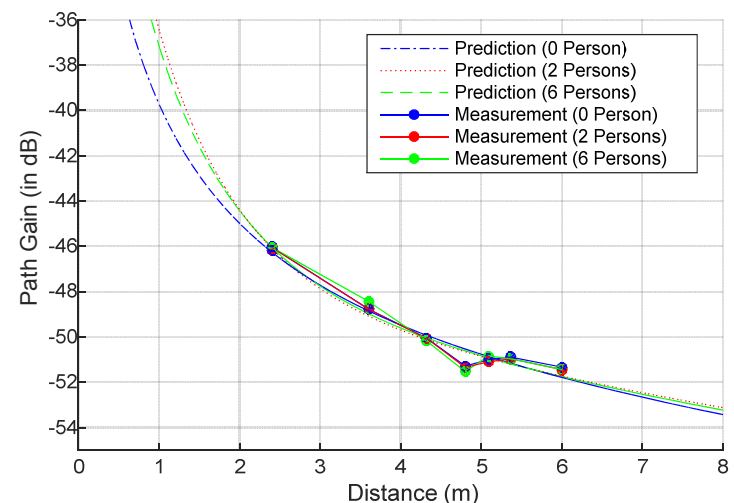

(a) Scenario $\mathrm{A}$

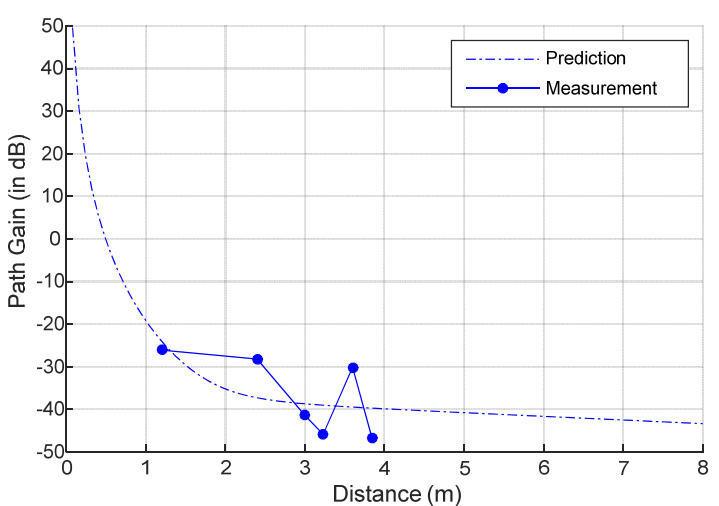

(b) Scenario B

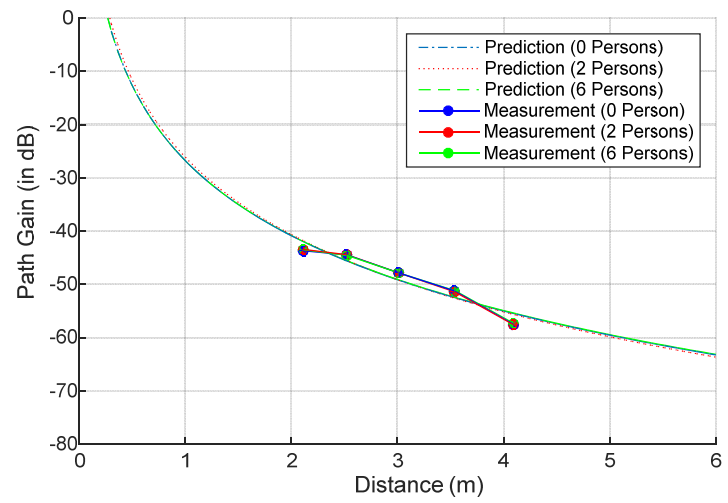

(c) Scenario C1 Up Direction

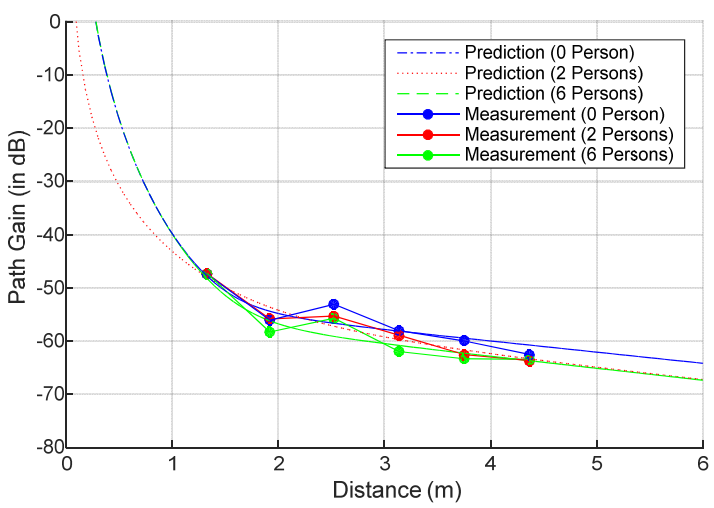

(d) Scenario C1 Down Direction

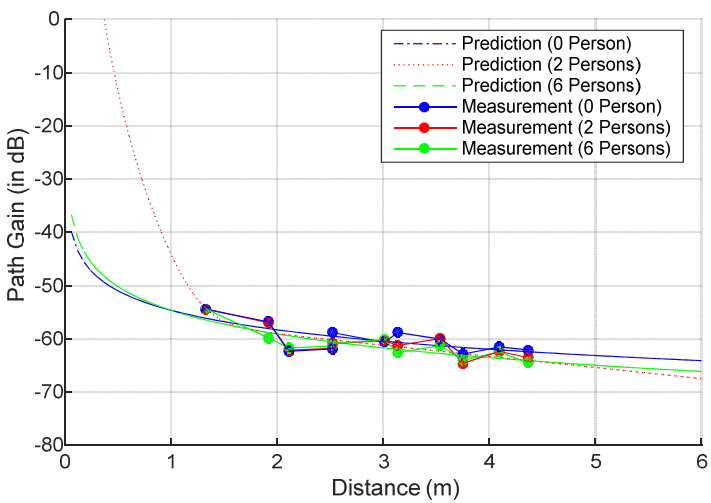

(e) Scenario C2

Fig. 6. Measured path gains and fitted curves.

\begin{tabular}{|c|c|c|}
\hline Parameters & Values & Description \\
\hline Scenario & $\begin{array}{l}\text { A, with } 0 \\
\text { persons }\end{array}$ & Scenario for validation \\
\hline Start point & $k=9$ & $\begin{array}{l}\text { To ensure that the first modelled bin is } \\
\text { later than the LOS component. }\end{array}$ \\
\hline$N$ & 38 & $\begin{array}{l}\text { Delays bins larger than } 200 \mathrm{~ns} \text { (i.e. } k>38 \text {, } \\
\text { as } \tau_{39}>200 \mathrm{~ns}>\tau_{38} \text { ) are not modelled. }\end{array}$ \\
\hline $\begin{array}{l}\text { Bin size } \\
\Delta \tau[\mathrm{ns}]\end{array}$ & 5.263 & $\begin{array}{l}\text { The bin size equals to the step size of } \\
\text { measured PDP delays. The latter is } \\
\text { dependent on the measurement system. }\end{array}$ \\
\hline$m_{\mu}$ & -0.074 & \multirow{2}{*}{$\begin{array}{l}\text { Obtained by log-normal fitting, as shown } \\
\text { in Fig. } 7 \text { (b). }\end{array}$} \\
\hline$\sigma_{\mu}$ & 0.199 & \\
\hline$B_{\omega}$ & -0.165 & \multirow{2}{*}{$\begin{array}{l}\text { Obtained by linear fitting, as shown in } \\
\text { Fig. } 7 \text { (c). }\end{array}$} \\
\hline$C$ & -3.658 & \\
\hline
\end{tabular}

Tab. 5. Data in use and model parameters.

Figure 7(a) provides the Nakagami fit of normalized amplitude. The log-normal distribution of shape parameter is illustrated in Fig. 7(b), and the quasi-linear decaying of scale parameter is shown in Fig. 7(c). The proposed DTDL model of PDP is obtained with these measured and fitted parameters. The model is presented in Fig. 7(d).

\section{Conclusions}

In this work, a novel model describing reverberation time and path gain in indoor wireless scenarios is proposed. The model is based on propagation geometry and materials of the scenarios and room electromagnetics. It takes both the reflections and effects along the propagation path into account. The new model is validated by the good agreement between predicted values and measurement results in $2.6 \mathrm{GHz}$ LOS scenarios, and it is proved to be more accurate than traditional models. PDP model is subsequently proposed based on measured path gain and Nakagami-m distribution. Complexity of PDP modeling is reduced compared to literature with the contribution of reverberation method. Also, the application of reverberation models is extended to semi-enclosed scenarios. The model is expected to be useful in the indoor wireless link budget, coverage prediction, and physical layer algorithms developments in future femto-cell-based wireless systems. 


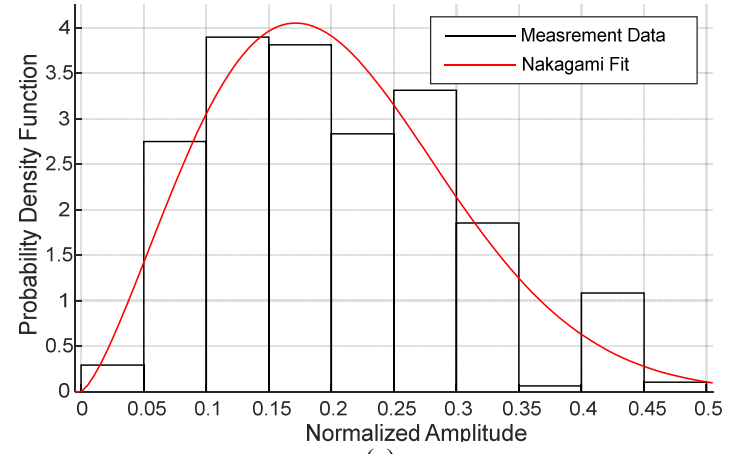

(a)

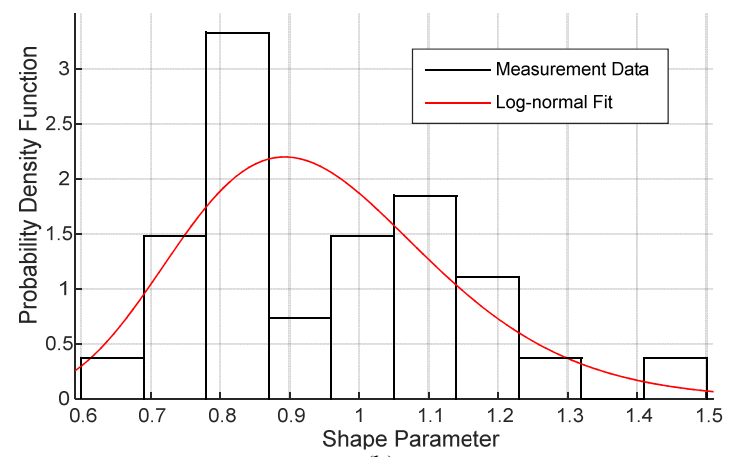

(b)

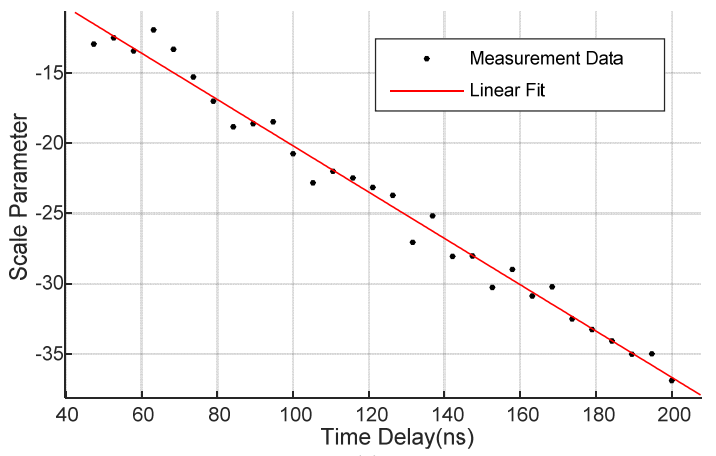

(c)

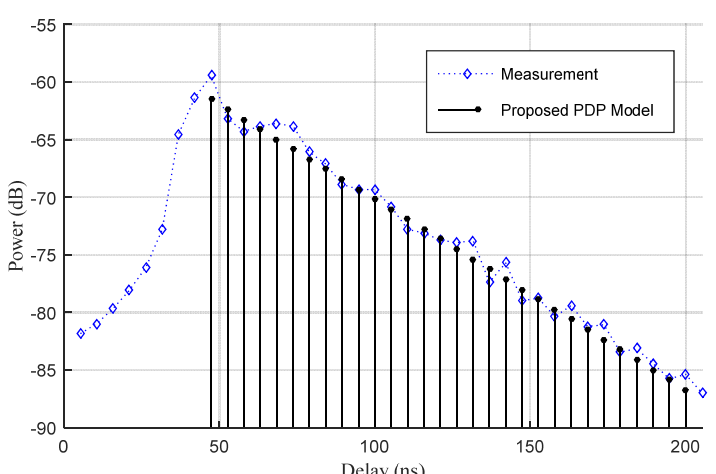

(d)

Fig. 7. The proposed PDP model.

\section{Acknowledgments}

This work is supported by the Natural Science Foundation of China (NSFC) under grants no. 61427801 and 61471204 .

\section{References}

[1] Andersen, J. B., NIELSEN, J. O., PEDERSEN, G. F., et al. Room electromagnetics. IEEE Antennas and Propagation Magazine, 2007, vol. 49, no. 2, p. 27-33. DOI: 10.1109/MAP.2007.376642

[2] ChEnG, S., GAillot, D. P., TANGHE, E., et al. Polarimetric distance-dependent models for large hall scenarios. IEEE Transactions on Antennas and Propagation, 2016, vol. 64, no. 5, p. 1907-1917. DOI: 10.1109/TAP.2016.2535100

[3] GAILlot, D. P., TANGHE, E., JOSEPH, W., et al. Polarization properties of specular and dense multipath components in a large industrial hall. IEEE Transactions on Antennas and Propagation, 2015, vol. 63, no. 7, p. 3219-3228. DOI: 10.1109/TAP.2015.2430374

[4] STEINBÖCK, G., PEDERSEN, T., FLEURY, B. H., et al. Distance dependent model for the delay power spectrum of inroom radio channels. IEEE Transactions on Antennas and Propagation, 2013, vol. 61, no. 8, p. 4327-4340. DOI: 10.1109/TAP.2013.2260513

[5] BAMBA, A., GAILlOT, D. P., TANGHE, E., et al. Experimental investigation of electromagnetic reverberation characteristics as a function of UWB frequencies. IEEE Antennas and Wireless Propagation Letters, 2015, vol. 14, p. 859-862. DOI: 10.1109/LAWP.2014.2382873

[6] BAMBA, A., JOSEPH, W., TANGHE, E., et al. Circuit model for diffuse multipath and electromagnetic absorption prediction in rooms. IEEE Transactions on Antennas and Propagation, 2013, vol. 61, no. 6, p. 3292-3301. DOI: 10.1109/TAP.2013.2250234

[7] NIELSEN, Ø., ANDERSEN, J. B., PEDERSEN, G. F., et al. On polarization and frequency dependence of diffuse indoor propagation. In 2011 IEEE Vehicular Technology Conference (VTC Fall). San Francisco (USA), 2011, p. 1-5. DOI: 10.1109/VETECF.2011.6092906

[8] YU, Y., LIU, Y., LU, W.-J., et al. Modelling and simulation of channel power delay profile under indoor stair environment. IET Communications, 2017, vol. 11, no. 1, p. 119-126. DOI: 10.1049/iet-com.2015.1004

[9] HOLlOWAY, C. L., COTTON, M. G., MCKENNA, P. A model for predicting the power delay profile characteristics inside a room. IEEE Transactions on Vehicular Technology, 1999, vol. 48, no. 4, p. 1110-1120. DOI: $10.1109 / 25.775360$

[10] STEINBÖCK, G., GAN, M., MEISSNER, P., et al. Hybrid model for reverberant indoor radio channels using rays and graphs. IEEE Transactions on Antennas and Propagation, 2016, vol. 64, no. 9, p. 4036-4048. DOI: 10.1109/TAP.2016.2589958

[11] SABINE, W. C. Collected Papers on Acoustics. Cambridge (MA, USA): Harvard Univ. Press, 1922.

[12] EYRING, C. F., Reverberation time in "dead" rooms. Journal of the Acoustical Society of America, 1930, vol. 1, no. 2, p. 217-241.

[13] KUTTRUFF, H. Room Acoustics. $4^{\text {th }}$ ed. New York (USA): Taylor \& Francis, 2000. ISBN: 9780419245803

[14] WENMAEKERS, R., HAK, C., HORNIKX, M. The effective air absorption coefficient for predicting reverberation time in full octave bands. Journal of the Acoustical Society of America, 2014, vol. 136 , no. 6 , p. 3063-3071. DOI: 10.1121/1.4901710

[15] INAN, S. U., INAN, S. A. Electromagnetic Waves. Englewood Cliffs (USA): Prentice-Hall, 2000. ISBN: 9780201361797

[16] BAMBA, A., JOSEPH, W., ANDERSEN, J. B., et al. Experimental assessment of specific absorption rate using room electromagnetics. IEEE Transactions on Electromagnetic Compatibility, 2012, vol. 54, no. 4, p. 747-757. DOI: 10.1109/TEMC.2012.2189572 
[17] CASSIOLI, D., WIN, M. Z., MOLISCH, A. F. The ultra-wide bandwidth indoor channel: from statistical model to simulations. IEEE Journal on Selected Areas in Communications, 2002, vol. 20, no. 6, p. 1247-1257. DOI: 10.1109/JSAC.2002.801228

[18] SHE, J., YANG, B., LIU, J., et al. Characterization of $2.6 \mathrm{GHz}$ wireless channel in meeting room scenario using reverberation theory. In IEEE International Conference on Ubiquitous Wireless Broadband (ICUWB). Nanjing (China), 2016, p. 1-4. DOI: 10.1109/ICUWB.2016.7790586

[19] WANG, Y., LU, W.-J., ZHU, H.-B. Propagation characteristics of the LTE indoor radio channel with persons at $2.6 \mathrm{GHz}$. IEEE Antennas and Wireless Propagation Letters, 2013, vol. 12, p. 991 to 994. DOI: 10.1109/LAWP.2013.2275811

[20] STEINBÖCK, G., PEDERSEN, T., FLEURY, B. H., et al. Experimental validation of the reverberation effect in room electromagnetics. IEEE Transactions on Antennas and Propagation, 2015, vol. 63, no. 5, p. 2041-2053. DOI: 10.1109/TAP.2015.2423636

[21] KUNISCH, J., PAMP, J. Measurement results and modeling aspects for the UWB radio channel. In Proceedings of IEEE Conference on Ultra Wideband Systems and Technologies. Baltimore (USA), 2002, p. 19-23. DOI: 10.1109/UWBST.2002.1006310

[22] PETER, M., KEUSGEN, W. Analysis and comparison of indoor wideband radio channels at 5 and $60 \mathrm{GHz}$. In Proceedings of the 3th European Conference on Antennas and Propagation (EuCAP'09). Berlin (Germany), 2009, p. 3830-3834.

\section{About the Authors ...}

Jun SHE was born in Nanjing, China, in 1989. He received the B.E. degrees in Electronic \& Information Engineering from Hohai University (HHU), Nanjing, China, and University of Lille 1 (USTL), Lille, France, in 2011. He received the M.S. degree in System of Communication in Radio-frequency from University of Lille 1 (USTL), Lille, France, in 2013. His research interests include wireless propagation and wireless channel modeling.

Yu YU was born in Nanjing, China, in 1990. He received the B.E. degree in Electrical Engineering from Nanjing University of Posts and Telecommunications (NUPT), Nanjing, China, in 2012. His research interests include wireless propagation and wireless channel modeling.
Peng-Fei CUI was born in Yancheng, China, in 1990. He received the B.E. degree in Electrical Engineering from Soochow University (SU), Suzhou, China, in 2013. His research interests include body centric communication, wireless channel measurement and channel modeling.

Wen-Jun LU was born in Jiangmen, China, in 1978. He received B.E. and Ph.D, degrees in Communication Engineering and Electrical Engineering from Nanjing University of Posts and Telecommunications (NUPT), Nanjing, China, in 2001 and 2007, respectively. He has been a Lecturer (2007-2009), an Associate Professor (2009-2013), and a Professor (since 2013) in Jiangsu Key Laboratory of Wireless Communications, NUPT. He has authored and co-authored over 100 technical papers published in peerreviewed international journals and conference proceedings. He is the translator of the Chinese version "The Art and Science of Ultra Wideband Antennas" (by H. Schantz). He authors the book "Antennas: Concise Theory, Design and Applications" (in Chinese). He has been serving as an editorial board member of International Journal of RF and Microwave Computer-Aided Engineering since 2014. He was the recipient of the Exceptional Reviewers Award of IEEE Transactions on Antennas and Propagation in 2016, Award of New Century Excellent Talents in Universities from the Ministry of Education of China in 2012, and the Nomination Award of Top-100 Outstanding PhD Dissertation of China in 2009. He was also the co-recipient of other 6 scientific and technological awards granted by the Jiangsu Province, Chinese Institute of Electronics, and Chinese Institute of Communications, respectively. His research interests include antenna theory, wideband antennas and arrays.

Hong-Bo ZHU (corresponding author) was born in Yangzhou, China, in 1956. He is presently the standing director of the Chinese Institute of Electronics and director of Jiangsu Key Laboratory of Wireless Communications, Nanjing University of Posts and Telecommunications (NUPT), Nanjing, China. He has authored and co-authored more than 100 journal papers and more than 60 invention patents authorized. In the past 5 years, he has undertaken more than 30 projects at the national, provincial and ministerial level. His research interests include wireless communications, Internet of Things (IoT) and EMC. 